\title{
A hybrid segmentation approach for geographic atrophy in fundus auto-fluorescence images for diagnosis of age-related macular degeneration.
}

\author{
Noah Lee, Andrew F. Laine, and R. Theodore Smith
}

\begin{abstract}
Fundus auto-fluorescence (FAF) images with hypo-fluorescence indicate geographic atrophy (GA) of the retinal pigment epithelium (RPE) in age-related macular degeneration (AMD). Manual quantification of GA is time consuming and prone to inter- and intra-observer variability. Automatic quantification is important for determining disease progression and facilitating clinical diagnosis of AMD. In this paper we describe a hybrid segmentation method for GA quantification by identifying hypo-fluorescent $G A$ regions from other interfering retinal vessel structures. First, we employ background illumination correction exploiting a non-linear adaptive smoothing operator. Then, we use the level set framework to perform segmentation of hypo-fluorescent areas. Finally, we present an energy function combining morphological scale-space analysis with a geometric model-based approach to perform segmentation refinement of false positive hypo- fluorescent areas due to interfering retinal structures. The clinically apparent areas of hypo-fluorescence were drawn by an expert grader and compared on a pixel by pixel basis to our segmentation results. The mean sensitivity and specificity of the ROC analysis were 0.89 and $0.98 \%$.
\end{abstract}

\section{INTRODUCTION}

Fundus auto-fluorescence (FAF) imaging is a non-invasive technique for in vivo ophthalmoscopic inspection of age-related macular degeneration (AMD), the most common cause of legal blindness in developed countries [3]. FAF signals are reliable markers of lipofuscin in retinal pigment epithelium (RPE) cells [1, 2]. Abnormally increased lipofuscin accumulations, which produce hyper-fluorescent FAF signals, occur in earlier stages of AMD. Geographic atrophy (GA) of the RPE, an advanced form of AMD, accounts for $12-21 \%$ of severe visual loss in this disorder [3]. GA is characterized by round or multi-lobed patches of atrophy of the RPE, the overlying retina, and the underlying choriocapillaris. With atrophy of the RPE, the lipofuscin is lost and the FAF signal from that region becomes hypo-fluorescent dark. Hypofluorescence is the FAF hallmark of geographic atrophy. A previous study suggests

Manuscript received April 16, 2007. This work was supported in part by NEI (R01 EY015520-01), the NYC Community Trust (RTS), and unrestricted funds from Research to prevent blindness.

Noah Lee is with the Heffner Biomedical Imaging Lab and the Department of Biomedical Engineering of Columbia University., CO 80305 USA (corresponding author to provide phone: 212-854-5996; fax: 212-854-5995; e-mail: n12168@columbia.edu).

Prof. A. Laine is with the Department of Biomedical Engineering, Columbia University, NY USA (e-mail: laine@columbia.edu).

Prof. R Theodore Smith is with the Department of Ophthalmology, Columbia University, NY, USA (e-mail: rts1@columbia.edu) that areas of increased FAF may precede development or enlargement of GA [4]. The left and middle panel of Fig. 1 illustrates this disease progression. Over time, atrophic patches may increase in size and number or may coalesce to form larger areas of atrophy. We seek to quantify atrophic changes by means of image analysis to provide measures for GA progression and to facilitate clinical diagnosis of GA in AMD.

Generally, GA quantification methods in the literature have typically relied on visual inspection of FAF images [13], which prevents quantification, or time consuming manual delineation of GA boundaries in combination with semi-automatic segmentation techniques [9]. Manual quantification of GA is time consuming and prone to interand intra-observer variability [13], especially for large patient studies over a period of time.

In this paper, we present a hybrid segmentation method exploiting non-linear smoothing operators for background illumination correction while preserving features of interest. We present an energy function combining morphological scale-space analysis with a geometric model-based approach to perform vessel likelihood estimation. Segmentation of GA is utilized within a level set framework and refined by our vessel likelihood estimates. We show quantitative results on GA border segmentation together with clinical evaluation and comparison to expert gradings.
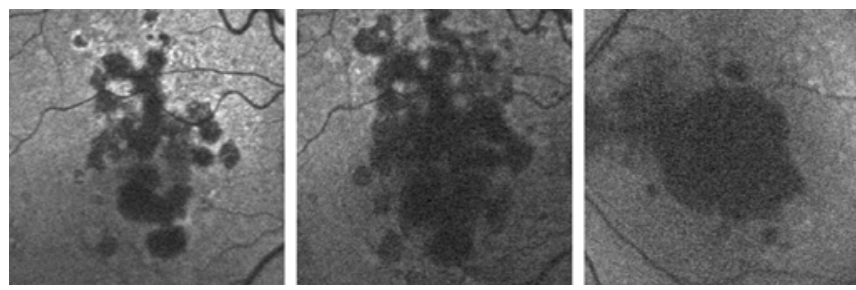

Fig. 1. FAF images and example of geographic atrophy (GA). The problem domain of non-homogenous background illumination and adjacent retinal vasculature. Left and middle image represent disease progression of GA over a period of 2-3 years. Bright areas surrounding the dark patches turn to dark areas within this period. Hypo-fluorescent dark areas indicate GA.

Little has been published on automated techniques for quantifying GA in FAF images. Recently, Deckert et al. proposed a region-growing method, where separate GA regions must be manually seeded to be included in the segmentation [10]. In a previous study, we have reported on a semi-automatic segmentation technique for GA quantification [9]. This work differs from our previous work in that we include three contributions: 1) background 
illumination correction by means of a non-linear smoothing operator, 2) segmentation of dark hypo-fluorescence areas using iterative fluorescence density minimization within the level set framework, and 3) vessel likelihood estimation using an energy function that combines morphological scale-space analysis with a geometric model-based approach for segmentation refinement. Regarding retinal background illumination correction, closely related to our approach are well known filtering based techniques such as homomorphic or median filtering. However, these approaches lack feature preservation as noted by Forachhia et al. [8]. In our work we exploit the bilateral filter [5] and show its suitability for luminosity estimation. For retinal vessel segmentation a vast literature can be found proposing different approaches. Our energy function is motivated by the work of Zana et al. [7] and the vesselness measure from Frangi et al. [12]. However, in our approach we only apply the hat-transform scale-space, since features of interest and noise have similar scales in FAF images due to small scale vasculature. We show that 3 ) provides robust vessel segmentation and prevents false positive vessel likelihood responses at GA border contour regions.

\section{MATERIALS AND Methodology}

FAF images of 14 eyes from seven patients with GA were selected retrospectively from a patient database imaged from 2002 to 2005 at the Columbia University. All eyes had drusen as well as GA. Each eye had an initial and a final FAF image representing a follow up of two to three years. The ages of the patients ranged from 76 to 82 years. After pupillary dilation, FAF images had been recorded using the Heidelberg model HRA confocal SLO (Heidelberg Inc, Heidelberg, DE). This instrument uses blue laser light at $488 \mathrm{~nm}$ for illumination and a barrier filter at $500 \mathrm{~nm}$ to limit the captured light to autofluorescent structures. The FAF images consisted of bit-mapped laser scans, 512 x 512 pixels in size, centered on the macula. Each image was an average of 3 to 6 scans composed by the SLO software. All images had a scale of approximately $15 \mu \mathrm{m}$ per pixel. The image was then cropped to a $6000 \mu \mathrm{m}$ square centered on the fovea. All subsequent processing were performed on these images.

\section{A. Background Illumination Correction}

We exploit the properties of the bilateral filter [5] to estimate background luminosity. Let a domain filter $S_{d}$ be

$$
\begin{aligned}
& S_{d}(p(\phi))=k_{d}^{-1}(\phi) \int c(\phi-y) \cdot(y) d y, \text { with } \\
& k_{d}(\phi)=\int c(\phi-y) d y
\end{aligned}
$$

Then $p(\phi)$ denotes auto-fluorescence density, $c$ the spatial kernel function, and $S_{d}(p(\phi))$ the low pass filtered auto-fluorescence output density. Similarly, a range filter can be defined as

$$
\begin{aligned}
& S_{r}(p(\phi))=k_{r}^{-1}(\phi) \int g(p(\phi)-p(y)) d y, \text { with } \\
& k_{r}(\phi)=\int g(p(\phi)-p(y)) d y .
\end{aligned}
$$

Here $g$ denotes the intensity kernel function, where kernel coefficients of $g$ are derived from auto-fluorescence density value differences between kernel center and the domain of the kernel neighborhood. Note that $S_{r}$ has no notion of geometric distance. Thus, in combination with $S_{d}$, smaller density value differences in $p(\phi)$ result in higher coefficient weights, leading to larger pixel contribution in $c$ irrespectively of geometric distance in the spatial kernel function. This property is suitable for luminosity estimation across high-frequency features such as GA border contour regions or retinal vessel structures. We note that other filtering based illumination correction techniques are not able to preserve high frequency density features [8]. Combining equation (1) and (2) is the bilateral filter

$$
S(p(\phi))=\frac{\int c(\phi-y) g(p(\phi)-p(y)) \cdot p(y) d y}{k_{d}(\phi) k_{r}(\phi)} .
$$

This combination enables diffusive smoothing across high frequency features, whereas other non-linear smoothing operators such as anisotropic diffusion [11] cannot smooth across edges. Consider the anisotropic diffusion equation of the auto-fluorescence density $f$

$$
\begin{aligned}
& \frac{\partial f}{\partial t}=\operatorname{div}\left(d\left(x_{1}, x_{2}, t\right) \nabla f\right), \text { with } \\
& d=e\left(\left\|\nabla f\left(x_{1}, x_{2}\right)\right\|\right)
\end{aligned}
$$

Looking at the diffusion coefficient $d$ of equation (4) we see that diffusion is prohibited across high frequency regions. However, in estimating luminosity distribution it is desirable to diffuse across high frequency features. Note that equation (3) enables diffusion across high frequency features due to the combination of spatial and intensity kernel functions $c$ and $g$. Following Tomasi et al. by using shift-invariant Gaussian kernels for $\mathrm{c}$ and $\mathrm{g}$, the filtered auto-fluorescence density $\mathrm{S}$ in discrete form is computed by single pass iteration

$$
\begin{aligned}
& S\left(p(\phi), \sigma_{d}, \sigma_{r}\right)= \\
& \frac{\sum_{i=-N}^{N} \sum_{j=-N}^{N} W \cdot f\left(p\left(\phi_{1}+i, \phi_{2}+j\right)\right)}{\sum_{i=-N}^{N} \sum_{j=-N}^{N} W} \text {, with }
\end{aligned}
$$




$$
\begin{aligned}
W & =W_{c}(\|p-\phi\|) \cdot W_{s}(|f(p)-f(\phi)|) \\
& =e^{-\frac{1}{2}\left(\frac{\|p-\phi\|}{\sigma_{d}}\right)^{2}} e^{-\frac{1}{2}\left(\frac{\|f(p)-f(\phi)\|}{\sigma_{r}}\right)^{2}}
\end{aligned}
$$

In words, by computing equation (5), we obtain the luminosity estimation of the auto-fluorescence density by selectively low-pass filtering regions with similar intensity ranges while preserving high-frequency density features such as border contours of GA regions. We estimate $W_{s}$ by the mean value difference of auto-fluorescence density peaks and specify $W_{c}$ based on anatomical assumptions of the largest appearing vessel diameter within our cropped $6000 \mu \mathrm{m}$ regions, i.e. $35 \mu \mathrm{m}$. The residual of luminosity estimation and the original auto-fluorescence density $f$ denotes the corrected illumination density distribution $S^{\prime}$, where

$$
S^{\prime}=f-S(p(\phi))-\left(N^{-1} \cdot \sum S(p(\phi))\right)
$$

\section{B. GA Segmentation using Level Set Framework}

We separate hypo-fluorescence areas from the background using an iterative density energy based minimization model. Let $S^{\prime}$ be the auto-fluorescence density in domain
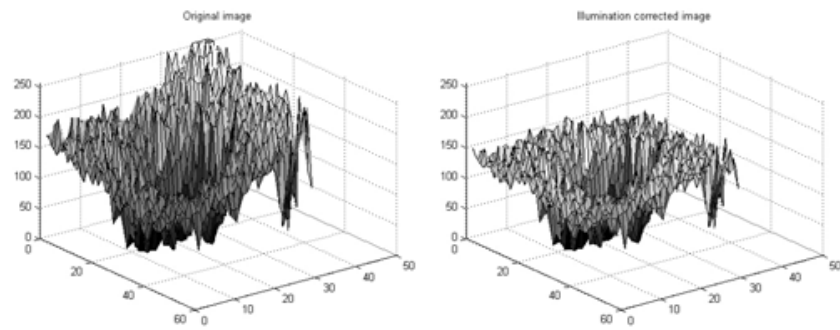

Fig. 2. Surface plot of background luminosity on FAF images. Left) Original background illumination, right) corrected background illumination using the bilateral filter.

$\Omega \in \mathfrak{R}^{2}$ with two homogenous regions of distinct density mean values $\mathrm{c} 1$ and $c_{2}$, where $c_{1}$ corresponds to the object region inside GA contour $\partial C$ and $c_{2}$ its inverse. To separate object from background we minimize the Chan-Vese energy functional [6].

$$
\begin{aligned}
F\left(\left\{c_{i}\right\}, \partial C\right)= & \mu \cdot|\partial C|+\lambda_{1} \int_{\partial C_{\text {out }}}\left|S^{\prime}-c_{1}\right|^{2} d \Omega \\
& +\lambda_{2} \int_{\partial C_{\text {in }}}\left|S^{\prime}-c_{2}\right|^{2} d \Omega
\end{aligned}
$$

Here $\mu, \lambda_{1}$, and $\lambda_{2}$ are weighting parameters for the individual fitting terms. In our implementation we used $\mu=0.03, \lambda_{1}=\lambda_{2}=1$ for all images and regularized versions of $H(\phi)$ and $\delta(\phi)$ as defined in [6]. The strength of the energy functional lies in the regularization term $\mu \cdot|\partial C|$, which acts as a smoothness constraint on $\partial C$. Parameter $\mu$ enforces curvature constraints during interface evolution, which prevents leaking into hypo-fluorescent vessel regions adjacent to GA regions. We use the level set framework to minimize equation (7), formulated as

$$
\begin{aligned}
F\left(\left\{c_{i}\right\}, \phi\right)= & \mu \int_{\Omega}\left|\nabla H_{\varepsilon}(\phi)\right| d \Omega \\
& +\lambda_{1} \int_{\Omega}\left|S^{\prime}-c_{1}\right|^{2} H_{\varepsilon}(\phi) d \Omega \\
& +\lambda_{2} \int_{\Omega}\left|S^{\prime}-c_{2}\right|^{2}\left(1-H_{\varepsilon}(\phi)\right) d \Omega
\end{aligned}
$$

Where the Heaviside function $H_{\varepsilon}(\phi)$ equals 1 if $\phi \geq 0$ and equals 0 if $\phi<0$. Due to regularization of $H(\phi)$ and $\delta(\phi)$ automatic arbitrary initialization of the initial zero level set contour is possible and no user interaction for initial contour definition is required. The functions $H_{\varepsilon}(\phi)$ and $\delta_{\varepsilon}(\phi)$ were approximated with $\varepsilon=2$. Rewriting in terms of $H_{\varepsilon}(\phi)$ transforms equation (7) into a problem of a single variable, which we minimize via gradient descent. Minimizing equation (8), the solution we obtain consists of a global minimum $F$ that includes over segmented parts such as hypo-fluorescent regions including the retinal vasculature.

\section{Energy Function for Vessel Likelihood Estimation}

To detect the vasculature we combine morphological scale space analysis with a multi-scale geometric model-based approach for vessel likelihood estimation using Frangi's vesselness measure. With $S^{\prime}$ being the auto-fluorescence density, we build a vessel likelihood map by computing the bottom-hat transform scale-space on $S^{\prime}$ with a structuring element $K_{\sigma_{1}}=\{3,5,7\}$.

$$
\begin{aligned}
& V_{h}\left(\phi,\left\{\sigma_{i}\right\}\right)=\int_{\Omega}\left(\sigma_{2}\right)^{2} \cdot H_{\sigma_{2}}\left\{G(\phi) \cdot W\left(\sigma_{2}\right)\right\} d \Omega . \\
& G(\phi)=\sum_{\sigma_{1}}^{n} S^{\prime}(\phi)-\left(S^{\prime}(\phi) \bullet K_{\sigma_{1}}\right)
\end{aligned}
$$

Here $H_{\sigma_{2}}$ denotes the Hessian matrix at scales $\sigma_{2}=\sigma_{1}, W$ is the second partial derivative of a Gaussian, $\sigma_{i}$ the scale parameters, and the summation term represents the bottom-hat transform scale space. We normalize $V_{h}$ by eigen 
analysis of $H_{\sigma_{2}}$ as defined in [12], where the normalized vessel likelihood is $V_{h}(\phi)=\max \left\{V_{h}\left(\phi,\left\{\sigma_{2}\right\}\right)\right\}$. The weighted combination of both feature responses introduces a penalty energy, where the vesselness measure alone produces false positive responses at GA border contour regions as shown in the following figure (right).
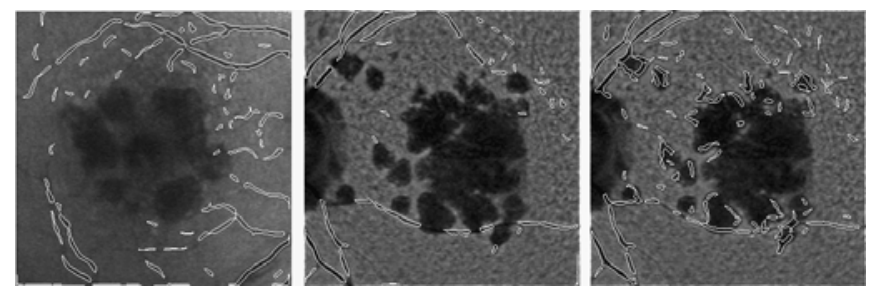

Fig. 3. Qualitative vessel segmentation performance. Left, middle) Results obtained with our energy functional combining morphological scale space analysis and geometric model-based vessel information. Right) Problem domain of model-based approach only at large GA regions.

This combination enables accurate vessel segmentation and resolves the problem of high vessel responses near GA areas. The hypo-fluorescent areas of the retinal vasculature were classified from the background using unsupervised k-means clustering with $\mathrm{k}=2$.

\section{RESULTS}

To validate our method we have selected a set of 14 FAF images comprising different levels of segmentation complexity in contrast and noise level. We performed validation compared to clinical expert grading and obtained a mean sensitivity of 0.89 and a mean specificity of 0.98 with standard deviations of \pm 0.09 and \pm 0.02 respectively. Seven patients had serial FAF imaging over a period of 2-3 years. For all experiments we used the parameter settings specified in Section 2. Ground truths and segmentation results were compared on a pixel-by-pixel basis using ROC analysis. Figure 4 shows final segmentation results of our method.

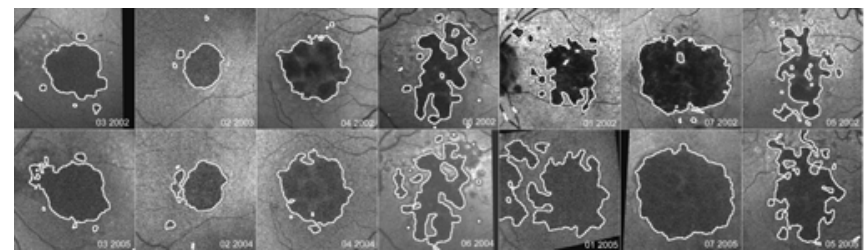

Fig. 4. Segmentation results on all 14 images. Each column represents patient FAF imaging (initial and final image) taken over a period of 2-3 years. Segmentation boundary overlaid onto the image data in white.

\section{CONCLUSION}

We have presented a method for GA segmentation in FAF images. We validated our method with the evaluation of clinical expert gradings. Results are promising indicating that accurate quantification of GA can be performed automatically on a variety of GA disorders. Extension to quantification of atrophic areas in FAF images of other retinal disorders also seems likely. Future research is intended towards parameter estimation for robust algorithm initialization and incorporation of higher level prior information into the segmentation process. Evaluation of our algorithm on large clinical datasets is planned as a next step.

\section{ACKNOWLEDGMENT}

This work was founded by NEI (R01 EY015520-01), the NYC Community Trust (RTS), and unrestricted funds from Research to Prevent Blindness.

\section{REFERENCES}

[1] Delori F.C., Fleckner M.R., Goger D.G. et al.: Auto-fluorescence distribution associated with drusen in age-related macular degeneration. Invest Ophthalmol Vis Sci. (2000); Vol. 41; 496-504.

[2] Delori F.C., Dorey C.K., Staurenghi G. et al.: In vivo fluorescence of the ocular fundus exhib-its retinal pigment epithelium lipofuscin characteristics. Invest Ophthalmol Vis Sci. (1995); Vol. 36; 718-29.

[3] Sunness J.S.: The natural history of geographic atrophy, the advanced atrophic form of age-related macular degeneration. Mol Vis. (1999); Vol. 5; 25-9.

[4] Holz F.G., Bellmann C., Staudt S. et al.: Fundus auto-fluorescence and development of geo-graphic atrophy in age-related macular degeneration. Invest Ophthalmol Vis Sci. (2001); Vol. 42; 1051-6.

[5] Tomasi C., Manduchi R.: Bilateral filtering for gray and color images. ICCV. (1998); 839-46.

[6] Chan T. F., Vese L. A.: Active Contours Without Edges. IEEE Transaction on Image Processing. (2001); Vol. 10; No. 2; 266-77.

[7] Zana F., Klein J.C.: Segmentation of Vessel-Like Patterns using Mathematical Morphology and Curvature Evaluation. IEEE Transaction on Image Processing. (2001); Vol. 10; No. 7; 1010-19.

[8] Foracchia M., Grisan E., Ruggeri A.: Luminosity and contrast normalization in retinal images. Elsevier Medical Image Analysis. (2004); Vol. 9; 179-90.

[9] Hwang J. C., Chan J. W. K., Chang S. et al.: Predictive Value of Fundus Auto-fluorescence for Development of Geographic Atrophy in Age-Related Macular Degeneration. Invest Ophthalmol Vis Sci. (2006); in press.

[10] Deckert A., Schmitz-Valckenberg S., Jorzik J. et al.: Automated analysis of digital fundus auto-fluorescence images of geographic atrophy in advanced age-related macular degeneration using confocal scanning laser ophthalmoscopy (cSLO). BME Ophthalmology. (2005); Vol 5.

[11] Perona P., Malik J.: Scale-Space and Edge Detection Using Anisotropic Diffusion. IEEE Trans. PAMI. (1990); Vol. 12, No. 7; 629.

[12] Frangi A.F., Niessen W.J., Nederkoorn P.J. et al.: Three-Dimensional Model-Based Stenosis Quantification of the Carotid Arteries from Contrast-Enhanced MR Angiography. IEEE Mathematical Methods in Biomedical Image Analysis. (2000); 110-8.

[13] Bindewald A., Bird A.C., Dandekar S.S. et al.: Classification of Fundus Auto-fluorescence Patterns in Early Age-Related Macular Disease. Invest. Ophthal Vis Sci. (2005); Vol. 45; 3309-14. 